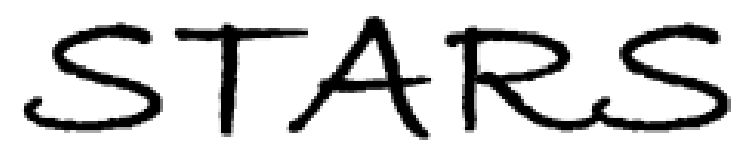

University of Central Florida

STARS

$1-1-2013$

\title{
Low temperature electron transport spectroscopy of mechanically templated carbon nanotube single electron transistors
}

\author{
Paul Stokes \\ University of Central Florida \\ Muhammad R. Islam \\ University of Central Florida \\ Saiful I. Khondaker \\ University of Central Florida
}

Find similar works at: https://stars.library.ucf.edu/facultybib2010

University of Central Florida Libraries http://library.ucf.edu

This Article is brought to you for free and open access by the Faculty Bibliography at STARS. It has been accepted for inclusion in Faculty Bibliography 2010 s by an authorized administrator of STARS. For more information, please contactSTARS@ucf.edu.

\section{Recommended Citation}

Stokes, Paul; Islam, Muhammad R.; and Khondaker, Saiful I., "Low temperature electron transport spectroscopy of mechanically templated carbon nanotube single electron transistors" (2013). Faculty Bibliography 2010s. 4714.

https://stars.library.ucf.edu/facultybib2010/4714

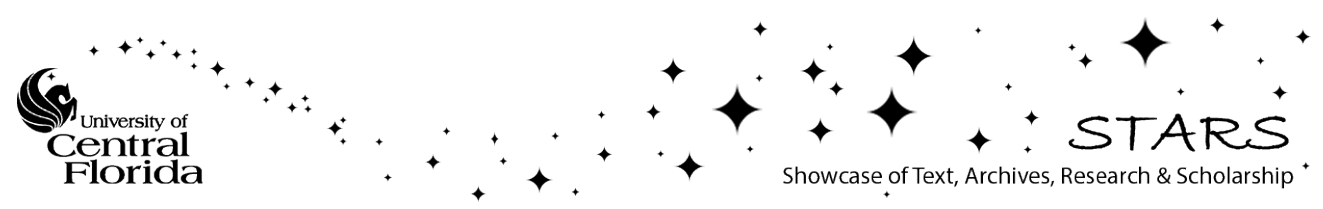




\section{Low temperature electron transport spectroscopy of mechanically templated carbon nanotube single electron transistors}

Cite as: J. Appl. Phys. 114, 084311 (2013); https://doi.org/10.1063/1.4819803

Submitted: 03 July 2013. Accepted: 14 August 2013 . Published Online: 29 August 2013

Paul Stokes, Muhammad R. Islam, and Saiful I. Khondaker
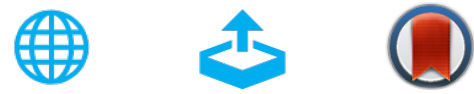

View Online

Export Citation

CrossMark

\section{ARTICLES YOU MAY BE INTERESTED IN}

The effect of carbon nanotube/organic semiconductor interfacial area on the performance of organic transistors

Applied Physics Letters 101, 233302 (2012); https://doi.org/10.1063/1.4769439

High quality solution processed carbon nanotube transistors assembled by dielectrophoresis Applied Physics Letters 96, 083110 (2010); https://doi.org/10.1063/1.3327521

Controlled fabrication of single electron transistors from single-walled carbon nanotubes Applied Physics Letters 92, 262107 (2008); https://doi.org/10.1063/1.2955520

\section{Applied Physics Reviews} Now accepting original research

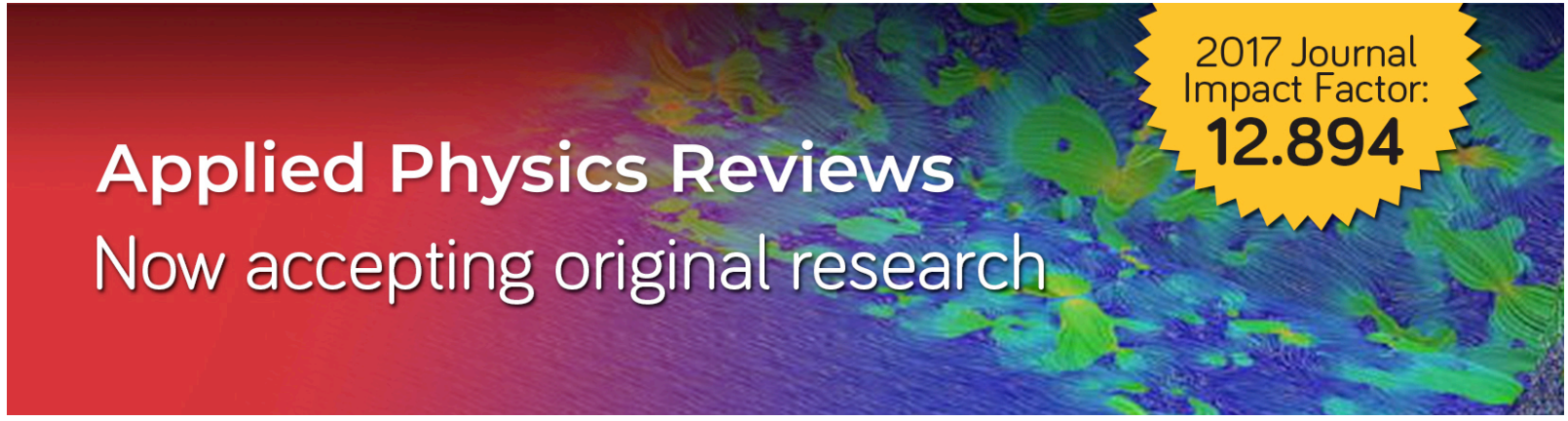




\title{
Low temperature electron transport spectroscopy of mechanically templated carbon nanotube single electron transistors
}

\author{
Paul Stokes, ${ }^{1,2}$ Muhammad R. Islam, ${ }^{1,2}$ and Saiful I. Khondaker ${ }^{1,2,3, a)}$ \\ ${ }^{1}$ Nanoscience Technology Center, University of Central Florida, Orlando, Florida 32826, USA \\ ${ }^{2}$ Department of Physics, University of Central Florida, Orlando, Florida 32826, USA \\ ${ }^{3}$ School of Electrical Engineering and Computer Science, University of Central Florida, Orlando, \\ Florida 32826, USA
}

(Received 3 July 2013; accepted 14 August 2013; published online 29 August 2013)

\begin{abstract}
We report electronic transport investigations of mechanically templated carbon nanotube single electron transistors (SETs). The devices were fabricated on a $\mathrm{Si} / \mathrm{SiO}_{2}$ substrate by controllably placing individual single walled carbon nanotubes (SWNTs) between the source and drain electrodes via dielectrophoresis with a $100 \mathrm{~nm}$ wide local $\mathrm{Al} / \mathrm{Al}_{2} \mathrm{O}_{3}$ bottom gate in the middle. From the low temperature electronic transport measurements, we show that a quantum dot is formed whose charging energy can be tuned from 10 to $90 \mathrm{meV}$ by varying both the local gate and $\mathrm{Si}$ backgate. The temperature dependent measurements show that the Coulomb oscillations persist up to $250 \mathrm{~K}$. The transport properties can be explained by a simple potential configuration, which suggests that two tunnel barriers are formed due to the bending of the SWNT at the local gate edges and that the size of the dot and tunnel barrier transparency can be tuned by the gates allowing the operation of SET in a wide temperature range and thereby realizing a controllable and tunable SET. Our simple fabrication technique and its tunability over a large temperature range could facilitate large scale fabrication of SET for practical applications. ( 2013 AIP Publishing LLC.

[http://dx.doi.org/10.1063/1.4819803]
\end{abstract}

\section{INTRODUCTION}

Single electron transistors (SETs) have attracted considerable attention because of their potential for low powered quantum based nanoelectronic devices such as ultrasensitive sensors, memory and quantum information processing. ${ }^{1-4} \mathrm{~A}$ SET consists of a small conducting island connected to two metallic leads through tunnel barriers. Electron tunneling can be controlled one by one with a nearby gate electrode, capacitively coupled to the island when its charging energy $\left(e^{2} / C\right)$ is greater than the thermal energy $\left(k_{B} T\right)$. By reducing the size of the island, the capacitance decreases leading to a higher charging energy and operating temperature. For the practical realization of SET based devices, controllable and reproducible fabrication of SET is required. Since the first SET was demonstrated about 25 years ago in an aluminum tunnel junction, ${ }^{5}$ it has been realized in a variety of systems including lithographically defined dots in GaAs/AlGaAs heterojunction, direct etching of $\mathrm{Si}$ substrate, metallic grains in nanopore, colloidal nanocrystals, nanowires, and nanotubes. ${ }^{6-15}$ Lithography defined dots are often larger in size, requiring sub-Kelvin temperature for operation. In addition, complex fabrication processes make it difficult to control the uniformity and reproducibility. Metallic grains and colloidal nanocrystals give smaller and more uniform quantum dots (QDs) with operating temperature $\sim 100 \mathrm{~K}$. However, fabrication of such devices is very challenging due

\footnotetext{
a) Author to whom correspondence should be addressed. Electronic mail: saiful@mail.ucf.edu
}

to difficulty in placement in nanosized gaps, hence leading to extremely low device yield.

Recently, single walled carbon nanotubes (SWNTs) ${ }^{16-25}$ have been considered to be a good candidate for the fabrication of SETs because of their extraordinary electronic properties, extremely small diameter along with unique one dimensional nature. Controllable and reproducible fabrication of SET using SWNT relies on the introduction of tunnel barriers at desired positions along the nanotubes so that charges can be confined in a quantum dot defined by the tunnel barriers. Metal contact induced Schottky barrier has been used to define SWNT based SET. ${ }^{22-25}$ However, the fabrication of SET using this technique is a "hit or miss" and controllability has yet to be demonstrated. It has also been shown that when a SWNT is bent at a selected position, the bend acts as a nanometer sized tunnel barrier. ${ }^{19-21}$ By creating a pair of bends on an individual SWNT using atomic force microscopy (AFM) tip, SETs have been demonstrated. ${ }^{19,20}$ However, AFM manipulation is time consuming and reproducibility of the same sized device can be challenging.

In order to circumvent this and create more controllable and reproducible SETs, we have recently introduced a simple technique to bend SWNT by placing it on top of a local $\mathrm{Al} / \mathrm{Al}_{2} \mathrm{O}_{3}$ gate between the source and drain contact. ${ }^{26}$ The local $\mathrm{Al} / \mathrm{Al}_{2} \mathrm{O}_{3}$ gate acts as a "mechanical template" and we hypothesized that SWNT bent at the edges of the local gate due to van der Walls interactions with the substrate to introduce two tunnel barriers. ${ }^{27}$ As a result, a QD is defined by the width of the local gate. Inducing tunnel barriers to the SWNT using this scheme can be advantageous over other 
techniques because of its simplicity of design and compatibility with large scale fabrication.

In this paper, we investigate the mechanisms for the tunnel barrier formation, its tunability, the role of the local gate for controlling the tunnel barrier and the SET, and demonstrate operation at higher temperature. The device is fabricated on $\mathrm{Si} / \mathrm{SiO}_{2}$ substrate by controllably placing individual single walled nanotube (SWNT) between the source and drain electrodes via dielectrophoresis (DEP) with a $100 \mathrm{~nm}$ wide local $\mathrm{Al} / \mathrm{Al}_{2} \mathrm{O}_{3}$ gate in the middle. We measured current oscillations at low temperature (down to $4 \mathrm{~K}$ ) as a function of local gate voltage for different Si back-gate voltages. We show that the charging energy of the SET can be tuned from 10 to $90 \mathrm{meV}$ by tuning both the gates. In addition, the temperature dependent measurements show that the single electron oscillations persist up to $250 \mathrm{~K}$ in qualitative agreement with the charging energy calculation. We also extract different device parameters from the slope of Coulomb diamond and explain the transport properties by a simple potential configuration, which suggests that two tunnel barriers are indeed formed due to the bending of the SWNT at the local gate edges and that the size of the dot and tunnel barrier transparency can be tuned by the gates allowing the operation of SET in a wide temperature range and thereby realizing a controllable and tunable SET. Our simple fabrication technique and its tunability over a large temperature range could facilitate large scale fabrication of SET for practical applications.

\section{EXPERIMENTAL DETAILS}

Figure 1(a) shows a schematic diagram of our device. The devices were fabricated on highly doped Si substrates with a thermally grown $250 \mathrm{~nm}$ thick $\mathrm{SiO}_{2}$ caped layer. Optical lithography was used to define contact pads and position alignment markers using double layer resists (LOR3A/ Shipley 1813), developed in CD26, followed by thermal evaporation of $5 \mathrm{~nm}$ chromium $(\mathrm{Cr})$ and $45 \mathrm{~nm}$ gold and then standard lift-off. The source and drain electrodes with a
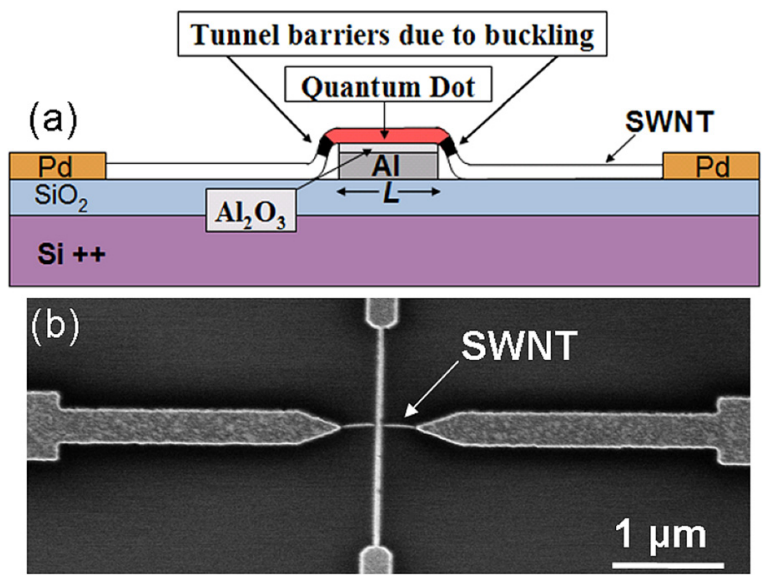

FIG. 1. (a) Schematic diagram of the mechanical template based SWNT SET device. (b) SEM of a fabricated device. An individual SWNT was placed over the local gate and between the source and drain electrodes by dielectrophoresis. The width of the local gate electrode is $\sim 100 \mathrm{~nm}$. Reprinted with permission from P. Stokes and S. I. Khondaker, Appl. Phys. Lett. 92, 262107 (2008). Copyright 2008 American Institute of Physics. channel length of $1 \mu \mathrm{m}$ were defined using standard electron beam lithography (EBL). After developing in (1:3) methyl isobutyl ketone:isopropyl alcohol (MIBK: IPA), $2 \mathrm{~nm} \mathrm{Cr}$ and $25 \mathrm{~nm}$ thick Pd were deposited by electron beam evaporation followed by lift-off in acetone. We used tapered shaped electrodes as they maximize the electric field at the sharp edge, which increases individual SWNT assembly yield. A second EBL was then performed to define a $100 \mathrm{~nm}$ wide local $\mathrm{Al}$ gate electrode of $25 \mathrm{~nm}$ thickness centered between the source and drain electrodes. During the Al deposition, the sample stage was cooled with liquid nitrogen to minimize the surface roughness of the $\mathrm{Al}^{28}$ Finally, the sample was treated in oxygen plasma for $10 \mathrm{~min}$ to ensure a $2-3 \mathrm{~nm}$ thick aluminum oxide $\left(\mathrm{Al}_{2} \mathrm{O}_{3}\right)$ layer.

The directed assembly of individual SWNT between the taper shaped electrode was done via DEP using a high quality aqueous solution of SWNT following our previous work. ${ }^{29-31}$ DEP has been used for the assembly of $2 \mathrm{D}, 1 \mathrm{D}$, and $0 \mathrm{D}$ nanomaterials for device applications. ${ }^{32-39} \mathrm{DEP}$ can be advantageous over other solution processed techniques because it allows the materials to be directly integrated to prefabricated electrodes. A stable, surfactant free solution with a concentration of SWNTs $\sim 50 \mu \mathrm{g} / \mathrm{ml}$ was obtained from Brewer Science. ${ }^{40}$ The average diameter of the SWNTs in the solution was $\sim 1.7 \mathrm{~nm}$ as determined by $\mathrm{AFM}^{41}$ For the assembly, the concentration of SWNTs was diluted to $\sim 10 \mathrm{ng} / \mathrm{ml}$ using DI water. A $3 \mu \mathrm{l}$ drop of solution was cast onto the chip and an $\mathrm{AC}$ voltage of $5 \mathrm{~V}_{\mathrm{p}-\mathrm{p}}$ at $1 \mathrm{MHz}$ was then applied between the source and drain electrodes. After $3 \mathrm{~min}$, the $\mathrm{AC}$ voltage was turned off and the remaining solution was blown off from the chip with a stream of nitrogen gas. A Zeiss Ultra 55 field emission SEM was used to examine the results of the DEP assembly. Figure 1(b) shows a representative scanning electron micrograph (SEM) image of an individual SWNT assembled between a source and drain electrode pair and on top of the local gate. The devices were then wire bonded and loaded into a ${ }^{4} \mathrm{He}$ cryostat for electronic transport measurements. DC charge transport measurements were performed by means of a DL instruments 1211 current preamplifier and a Keithley 2400 sourcemeter interfaced with LabView.

\section{RESULTS AND DISCUSSIONS}

Figure 2(a) shows a plot of room temperature drain current as a function of local gate $\left(V_{\mathrm{LG}}\right)$ and back gate voltages $\left(V_{\mathrm{BG}}\right)$ for one of our representative DEP assembled device. The highly doped $\mathrm{Si}$ was used as a back gate. The device shows a small change in current as a function of gate voltage indicating a small band gap SWNT. ${ }^{42}$ The detailed room temperature property of our DEP assembled devices can be found in our previous publications. ${ }^{29-32}$ In this paper, we only focus on low temperature SET behavior. It should be noted that the local gate shows more efficient gate coupling compared to the back gate due to thinner gate oxide with higher dielectric constant compared to the $\mathrm{SiO}_{2} \cdot{ }^{31}$

Figure 2(b) shows current versus $V_{\mathrm{LG}}$ for a fixed source drain voltage of $V_{D S}=5 \mathrm{mV}$ measured at a temperature (T) of $4.2 \mathrm{~K}$ and $V_{\mathrm{BG}}=0 \mathrm{~V}$. Current oscillations are observed as 

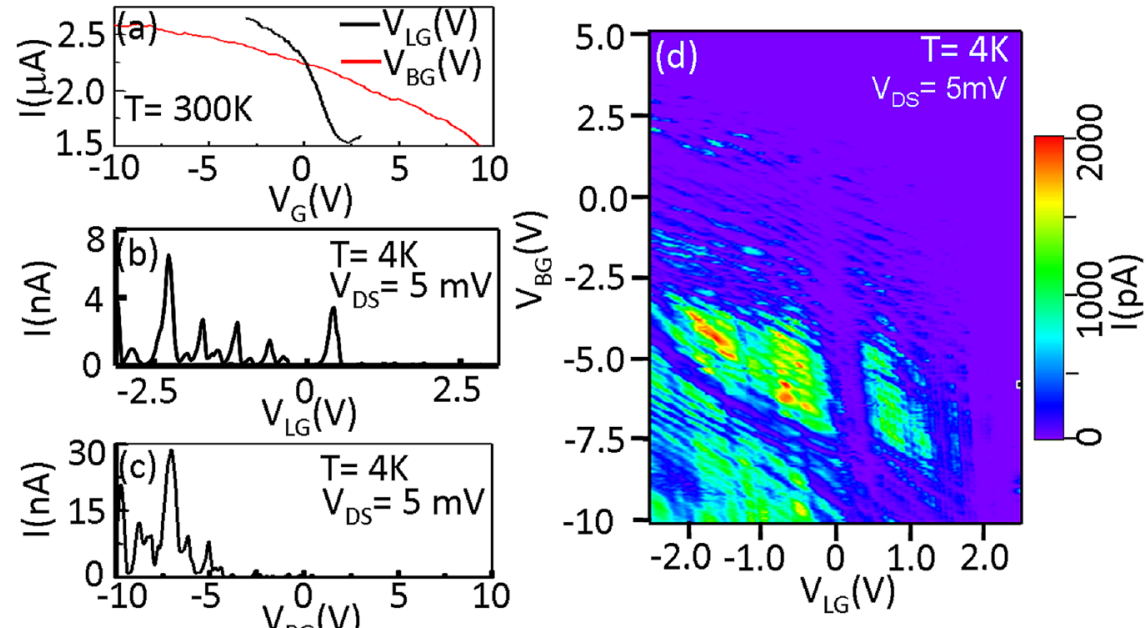

FIG. 2. (a) Current $(I)$ versus local gate voltage $\left(V_{L G}\right)$ and back gate voltage $\left(V_{B G}\right)$ of a representative device measured at room temperature. $V_{D S}=0.1 \mathrm{~V}$ for both curves. (b) $I$ versus $V_{L G}$ measured at T $=4.2 \mathrm{~K}$. $V_{B G}=0 \mathrm{~V}$ and $V_{D S}=5 \mathrm{mV}$. (c) $I$ versus $V_{B G}$ measured at T=4.2 K. $V_{L G}=0 \mathrm{~V}$ and $V_{D S}=5 \mathrm{mV}$. Current oscillations can be seen in (b) and (c), typical of a SET behavior. (d) Color scale plot of I versus $V_{B G}$ and $V_{L G}$ taken at $\mathrm{T}=4.2 \mathrm{~K}$ with a constant source-drain voltage of $5 \mathrm{mV}$. Bright regimes symbolize high current and dark regimes symbolize low current. Diagonal lines running throughout the plot indicating that the conductance of the device can be tuned by using both the local gate and the back gate.

a function of gate voltage, typical of SET behavior. ${ }^{43}$ Figure 2(c) shows a plot of current versus $V_{\mathrm{BG}}$ with $V_{\mathrm{LG}}=0 \mathrm{~V}$, and $V_{D S}=5 \mathrm{mV}$ at $\mathrm{T}=4.2 \mathrm{~K}$, showing similar SET behavior. The observation of SET behavior suggests that a quantum dot has formed in the channel and that the SET can be independently controlled by both the back gate and the local gate.

Figure 2(d) displays a color scale plot of current $(I)$ as a function of both $V_{\mathrm{BG}}$ and $V_{\mathrm{LG}}$ measured at $4.2 \mathrm{~K}$ at a fixed bias voltage $\left(V_{D S}=5 \mathrm{mV}\right)$. The current was measured by changing $V_{\mathrm{BG}}$ from -10 to $10 \mathrm{~V}$ at a step of $20 \mathrm{mV}$, while sweeping the local gate from -2.5 to $2.5 \mathrm{~V}$ at every $V_{\mathrm{BG}}$. The darker regions signify Coulomb Blockade, while other bright colors running diagonal to the figure signify peak of current oscillations. The alternating diagonal bright and dark lines demonstrate that, in addition to independent control of the SET by the gates, their combined effect can also control the operating regime of the SET. For example, for a $V_{\mathrm{BG}}$ of $-7.5 \mathrm{~V}$, the current oscillations persist for a $V_{\mathrm{LG}}$ of -2.5 to $2.5 \mathrm{~V}$, while for a $V_{\mathrm{BG}}$ of $+5 \mathrm{~V}$, there are no current oscillations for any value of $V_{\mathrm{LG}}$. In order to more clearly understand this combined effect of the back gate and local gate on our device, we present detailed analysis of our data at two constant back gate voltage regimes: (a) $V_{B G}=-7 \mathrm{~V}$ where the conductance is high, and (b) $V_{B G}=1.5 \mathrm{~V}$ where the conductance begins to die off. Our analysis is based on color scale plots of the differential conductance $\left(\mathrm{d} I / \mathrm{d} V_{D S}\right)$, as a function of $V_{\mathrm{DS}}$ and $V_{L G}$ at $4.2 \mathrm{~K}$ (often called a stability plot) to determine behavior of the device in these regimes. The stability plots allow us to determine the charging energy of the dot, various capacitances associated with the dot, as well as size of the dots at those regimes.

Figure 3(a) shows the stability plot with $V_{\mathrm{BG}}=-7 \mathrm{~V}$. The $V_{D S}$ was varied from -50 to $+50 \mathrm{mV}$ with a step of $0.5 \mathrm{mV}$, while the $V_{L G}$ was swept from -1.5 to $+1.5 \mathrm{~V}$. Brighter regions (red and white) symbolize high conductance and darker regions (blue) signify Coulomb blockade. Throughout the plot, a series of diamond shaped regions called Coulomb diamonds are observed. Closing of the diamonds indicates measurement of a single quantum dot. ${ }^{43}$ However, the height of the diamonds and their slopes changes with changing $V_{\mathrm{LG}}$. In order to investigate this in more detail, we focus on two local gate regions: $(I)$ $-1.25<V_{\mathrm{LG}}<-0.75 \mathrm{~V}$ and (II) $0.5<V_{\mathrm{LG}}<1.5 \mathrm{~V}$. These
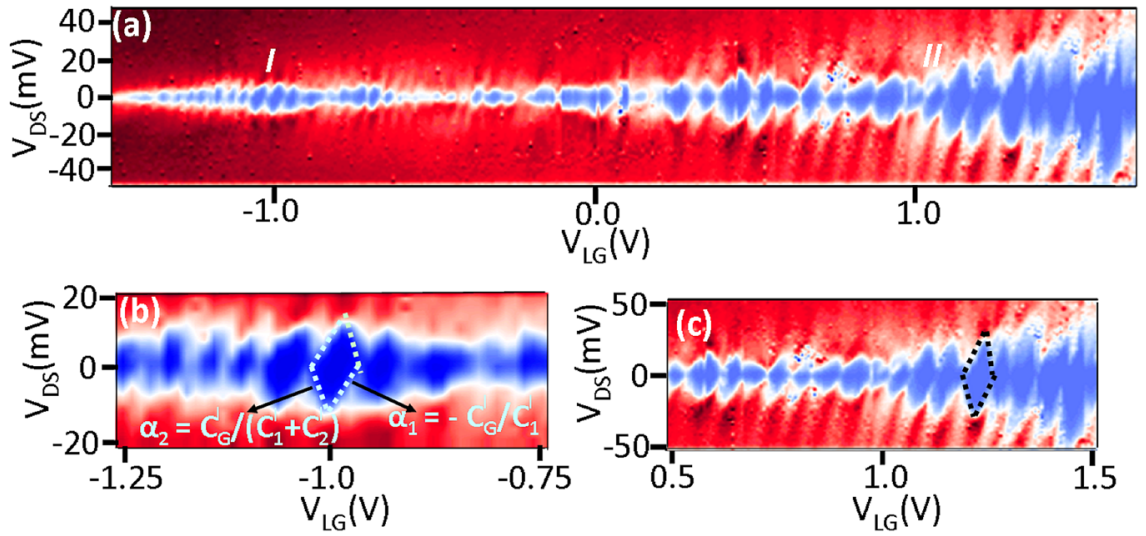

FIG. 3. (a) $\mathrm{d} / / \mathrm{d} V_{D S}$ versus $V_{\mathrm{DS}}$ and $\mathrm{V}_{\mathrm{LG}}$ taken at $4.2 \mathrm{~K}$ with $V_{\mathrm{BG}}=-7 \mathrm{~V}$. Red regions symbolize high conductance and blue regions symbolize low conductance or Coulomb blockade. (b) Expanded view of the stability plot in region $I$ showing transport through a single QD with charging energy $8-12 \mathrm{meV}$. (c) Expanded view of stability plot in region II showing single QD behavior with charging energy $\sim 20-45 \mathrm{meV}$. 


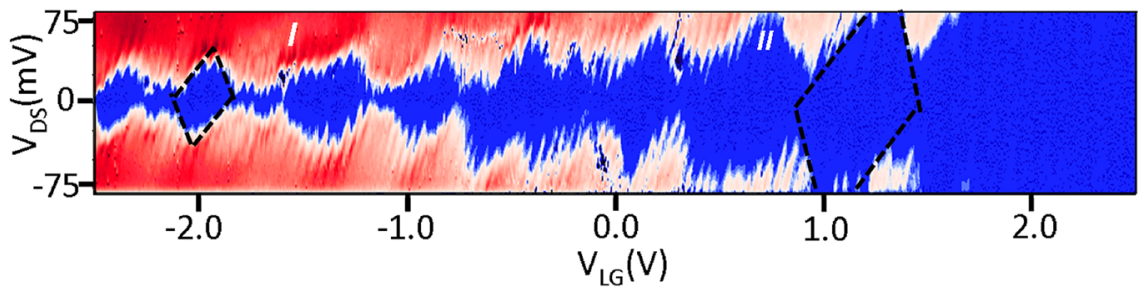

FIG. 4. $\mathrm{d} I / \mathrm{d} V_{D S}$ versus $V_{\mathrm{DS}}$ and $\mathrm{V}_{\mathrm{LG}}$ taken at $4.2 \mathrm{~K}$ with $V_{\mathrm{BG}}=1.5 \mathrm{~V}$. regions are expanded in Figures 3(b) and 3(c), respectively. The height of the diamond is a direct measure of charging energy $\left(U_{c}\right)$ of the quantum dot. The dashed lines in Fig. 3(b) are placed for a guide to the eye to outline a diamond from which we obtain $U_{c} \sim 10 \mathrm{meV}$. The capacitance values of the SET can be calculated from the Coulomb diamond using constant interaction model. ${ }^{43}$ The gate capacitance is calculated from the period $\left(\Delta V_{G}^{I}\right.$ $\sim 50 \mathrm{mV}$ ) between the diamonds using the formula $C_{G}^{I}=e / \Delta V_{G}^{I}$, which yields $C_{G}^{I} \sim 3 \mathrm{aF}$. The source and drain capacitances are calculated from the positive slope $\alpha_{1}=-C_{G}^{I} / C_{1}^{I}$ and negative slope $\alpha_{2}=C_{G}^{I} /\left(C_{1}^{I}+C_{2}^{I}\right)$ of the diamond, respectively (see Fig. 3(b)), which yield $C_{1}^{I}$ $\sim 12 \mathrm{aF}$ and $C_{2}^{I} \sim 1.5 \mathrm{aF}$. The total capacitance of the SET is $C_{\Sigma}^{I}=C_{1}^{I}+C_{2}^{I}+C_{G}^{I}=16.5 \mathrm{aF}$, which can be used to calculate the charging energy of $U_{C}^{I}=e^{2} / C_{\Sigma}^{I} \sim 10 \mathrm{meV}$ in good agreement with the value measured directly from the height of the diamond in this regime. It has been shown that the charging energy of a SWNT quantum dot can be estimated from the formula, $U_{C}=5 \mathrm{eV} / L(\mathrm{~nm})$, where $\mathrm{L}$ is the length the SWNT quantum dot in $\mathrm{nm}^{44}$ Using the value of $U_{c}=10 \mathrm{meV}$, we calculate a QD of size $\sim 500 \mathrm{~nm}$. Figure 3(c) shows an expanded view of the stability plot for $0.5<V_{\mathrm{LG}}$ $<1.5 \mathrm{~V}$ (region $I I)$. Here, the period is $\left(\Delta V_{G}^{I I} \sim 70 \mathrm{mV}\right)$ giving a gate capacitance $C_{G}^{I I} \sim 2.3 \mathrm{aF}$. The source and drain capacitances are $C_{1}^{I I} \sim 2.1 \mathrm{aF}$ and $C_{2}^{I I} \sim 0.35 \mathrm{aF}$, respectively, giving a total capacitance of $C_{\Sigma}^{I I}=C_{1}^{I I}+C_{2}^{I I}+C_{G}^{I I} \sim 4.8 \mathrm{aF}$ and a charging energy of $U_{C}^{I I}=e^{2} / C_{\Sigma}^{I I} \sim 34 \mathrm{meV}$, consistent with the height of the diamond in this regime. From here, we obtain a QD size of $\sim 147 \mathrm{~nm}$.

Figure 4 shows the stability plot of the same device at $V_{\mathrm{BG}}=1.5 \mathrm{~V}$. The charging energy in this case is substantially higher compared to $V_{\mathrm{BG}}=-7.0 \mathrm{~V}$, which is shown in Figure 3. The color scale plot exhibits a large diamond of spacings $\Delta V_{G} \sim 300-400 \mathrm{meV}$ inside which there is a periodic $\left(\Delta V_{G} \sim 50 \mathrm{meV}\right)$ saw tooth structure. This is a signature of charge transport through a small quantum dot in series with a large quantum dot; however, the smaller QD dominates the transport. ${ }^{45,46}$ The charging energy read off from the diamond is $\sim 40 \mathrm{meV}$ for region $I\left(-2.2<V_{\mathrm{LG}}<-0.75 \mathrm{~V}\right)$ and is

TABLE I. Summary of capacitance and charging energy values for the device at different local gate regions for positive and negative back gate voltages.

\begin{tabular}{lrcccccc}
\hline $\mathrm{V}_{\mathrm{BG}}(\mathrm{V})$ & Region & $\mathrm{C}_{\mathrm{G}}(\mathrm{aF})$ & $\mathrm{C}_{1}(\mathrm{aF})$ & $\mathrm{C}_{2}(\mathrm{aF})$ & $\mathrm{C}_{\Sigma}(\mathrm{aF})$ & $\mathrm{E}_{\mathrm{C}}(\mathrm{meV})$ & $\mathrm{L}(\mathrm{nm})$ \\
\hline-7 & $\mathrm{I}$ & 3 & 12 & 1.5 & 16.5 & 10 & 500 \\
-7 & $\mathrm{II}$ & 2.3 & 2.1 & 0.35 & 4.75 & 34 & 147 \\
1.5 & $\mathrm{I}$ & 0.6 & 2.6 & 0.9 & 4.1 & 40 & 125 \\
1.5 & $\mathrm{II}$ & 0.4 & 1.3 & 0.06 & 1.8 & 90 & 55 \\
\hline \hline
\end{tabular}

$\sim 90 \mathrm{meV}$ in region $I I\left(-0.75<V_{\mathrm{LG}}<1.5 \mathrm{~V}\right)$. This corresponds to a QD size of $125 \mathrm{~nm}$ in region $\mathrm{I}$ and $55 \mathrm{~nm}$ in region $I I$, respectively. The capacitances associated with the QD are calculated from the slope of the diamond and is presented in Table I, along with the capacitance, charging energy, and the corresponding size of QD for the regimes shown in Figures 3 and 4 . It can be seen that the charging energy increased from 10 to $90 \mathrm{eV}$ as the total capacitance decreased from 16.5 to $1.8 \mathrm{aF}$ under different $V_{B G}$ and $V_{L G}$ conditions demonstrating that the SET is highly tunable in charging energy.

Figure 5 shows the drain current of the SET versus the local gate voltage at different temperatures for fixed value of $V_{B G}=-7 \mathrm{~V}$ (Figure 5(a)) and $V_{B G}=1.5 \mathrm{~V}$ (Figure 5(b)). The bias voltage was kept fixed at $5 \mathrm{mV}$. For $V_{B G}=-7 \mathrm{~V}$, the current oscillations persist up to $120 \mathrm{~K}$, whereas for $V_{B G}=1.5 \mathrm{~V}$ the oscillations persist up to $250 \mathrm{~K}$. This shows that with increasing $V_{B G}$, the operating temperature increases, consistent with Figures 3 and 4, where we observed a larger charging energy with increasing $\mathrm{V}_{\mathrm{BG}}$. We can also estimate the charging energy of the quantum dot from the temperature dependent data using the formula, $U_{c}=4 K_{B} T^{47,48}$ For $\mathrm{T}=250 \mathrm{~K}, U_{c}=86 \mathrm{meV}$ which is close to the charging energy obtained from the stability plot.

The most important observation in our study is that the charging energy and operating temperature can be increased with increasing $V_{B G}$ and $V_{L G}$. From here, one can conclude that the system is highly tunable in charging energy and temperature. We have measured three other devices and found that this observation is typical for all of our devices. This is due to modification of tunnel barriers with gate voltage which in effect defines how the QDs are localized on the SWNT, which is evident in Table I. In order to understand this, we put forward a generalized potential configuration on

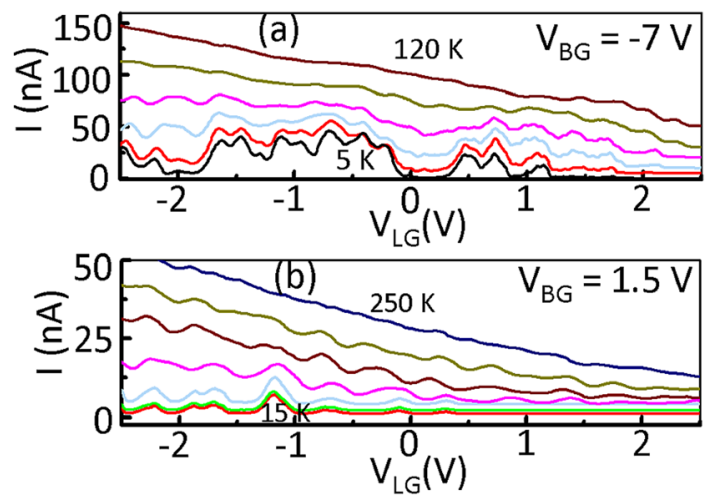

FIG. 5. Dependence of the drain current on local gate voltage at two different back gate voltages using $\mathrm{V}_{\mathrm{DS}}=5 \mathrm{mV}$. (a) Temperature dependent I versus $\mathrm{V}_{\mathrm{LG}}$ at a fixed $\mathrm{V}_{\mathrm{BG}}=-7 \mathrm{~V} . \mathrm{T}=5,15,30,50,80$, and 120 from bottom to top. (b) Temperature dependent $\mathrm{I}$ versus $\mathrm{V}_{\mathrm{LG}}$ at a fixed $\mathrm{V}_{\mathrm{BG}}=1.5 \mathrm{~V}$. $\mathrm{T}=15,30,50,80,120,200$, and $250 \mathrm{~K}$ (bottom to top). 


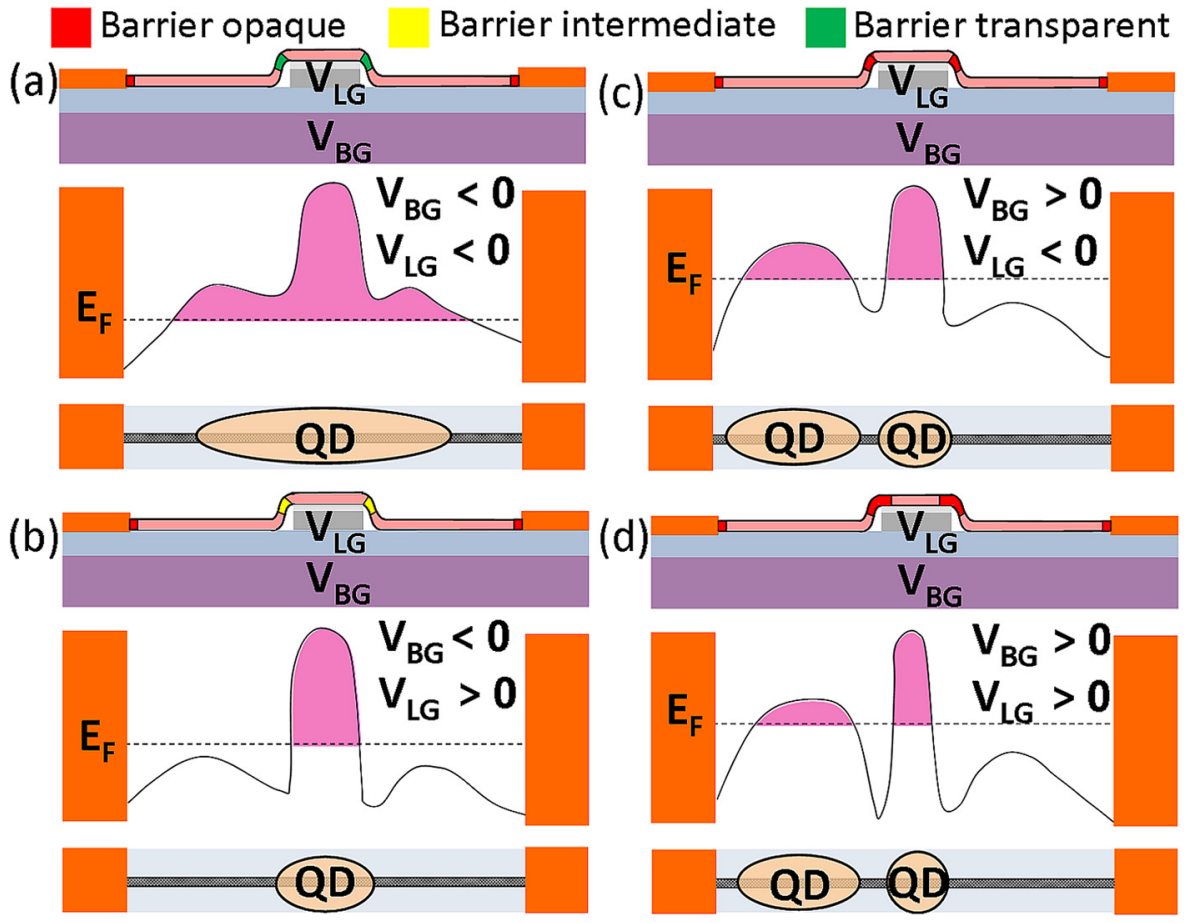

FIG. 6. Potential profile for the quantum dot formation at different local gate and back gate voltage regimes. how the tunnel barriers are formed by the mechanical templates and modified by the local and backgate voltages, shown in Figure 6.

We first discuss the case for $V_{B G} \ll 0 \mathrm{~V}, V_{L G} \ll 0 \mathrm{~V}$ (Figure 6(a)). In this case, the tunnel barriers defined by the local gate voltage are transparent and the QD is formed by the contact. This is consistent with the fact that the charging energies are small leading to a large QD. However, for $V_{B G}$ $\ll 0 \mathrm{~V}$ and $V_{L G} \gg 0 \mathrm{~V}$ (Figure 6(b)), the tunnel barriers caused by the local gate become opaque and all other potential fluctuations are placed below the Fermi level. As a result, the QD is defined by the local gate only as the QD size in this regime is close to the physical width of the local gate. Now we discuss the case where $V_{B G} \gg 0 \mathrm{~V}$ (right column of Figure 6). In this regime, the tunnel barriers defined by the local gate are opaque and the QD that dominates the transport is defined by the local gate. The observed beating may be due to another quantum dot formed by the charge fluctuations caused by the substrate. This leads to the formation of two QD, the small QD is defined by the local gate edge and the larger one by charge fluctuations. ${ }^{49}$ The increased charging energy with increasing $V_{L G}$ suggests that the tunnel barriers defined by the local gate gotten wider, further reducing the size of the dominant QD.

\section{CONCLUSION}

In conclusion, we studied the electronic transport properties of mechanically templated carbon nanotube SETs. The charging energy of the SET can be varied from 10 to $90 \mathrm{meV}$ by tuning the local gate and back gate voltages, which allowed the operating temperature to be up to $250 \mathrm{~K}$. The transport properties can be explained by a simple potential configuration, which suggests that the bending of the SWNT at the local gate edges creates two tunnel barriers and that the size of the dot and tunnel barrier transparency can be tuned by the gates allowing the operation of SET in a wide temperature range and thereby realizing a controllable and tunable SET. This simple fabrication technique along with tunability over a large temperature range could facilitate large scale fabrication of SET for practical applications.

\section{ACKNOWLEDGMENTS}

This work was supported by the U.S. National Science Foundation under Grant No. ECCS-0748091 (CAREER).

${ }^{1}$ M. H. Devoret and C. Glattli, Phys. World 11, 29 (1998).

${ }^{2}$ K. K. Likharev, Proc. IEEE 87, 606 (1999).

${ }^{3}$ R. H. Chen, A. N. Korotkov, and K. K. Likharev, Appl. Phys. Lett. 68, 1954 (1996)

${ }^{4}$ R. J. Schoelkopf, P. Wahlgren, A. A. Kozhevnikov, P. Delsing, and D. E. Prober, Science 280, 1238 (1998).

${ }^{5}$ T. A. Fulton and G. D. Dolan, Phys. Rev. Lett. 59, 109 (1987).

${ }^{6}$ J. A. Folk, S. R. Patel, S. F. Godijn, A. G. Huibers, S. M. Cronenwett, C. M. Marcus, K. Campman, and A. C. Gossard, Phys. Rev. Lett 76, 1699 (1996).

${ }^{7}$ Y. Takahashi, H. Namatsu, K. Kurihara, K. Iwdate, M. Nagase, and K. Murase, IEEE Trans. Electron Devices 43, 1213 (1996).

${ }^{8}$ K. I. Bolotin, F. Kuemmeth, A. N. Pasupathy, and D. C. Ralph, Appl. Phys. Lett. 84, 3154 (2004).

${ }^{9}$ D. Klein, R. Roth, A. K. L. Lim, A. P. Alivisatos, and P. L. McEuen, Nature (London) 389, 699 (1997).

${ }^{10}$ S. I. Khondaker, K. Luo, and Z. Yao, Nanotechnology 21, 095204 (2010).

${ }^{11}$ T. Mori, S. Sato, K. Omura, S. Yajima, Y. Tsuruoka, Y. Achiba, and K. Ishibashi, J. Vac. Sci. Technol. B 27, 795 (2009).

${ }^{12}$ K. Maehashi, H. Ozaki, Y. Ohno, K. Inoue, K. Matsumoto, S. Seki, and S. Tagawa, Appl. Phys. Lett. 90, 023103 (2007).

${ }^{13}$ M. Suzuki, K. Ishibashi, K. Toratani, D. Tsuya, and Y. Aoyagi, Appl. Phys. Lett. 81, 2273 (2002).

${ }^{14}$ M. T. Bjork, C. Thelander, A. E. Hansen, L. E. Jensen, M. W. Larsson, L. R. Wallenberg, and L. Samuelson, Nano Lett. 4, 1621 (2004).

${ }^{15}$ Z. Zhong, Y. Fang, W. Lu, and C. M. Lieber, Nano Lett. 5, 1143 (2005).

${ }^{16}$ M. Bockrath, D. H. Cobden, P. L. McEuen, N. G. Chopra, A. Zettl, A. Thess, and R. E. Smalley, Science 275, 1922 (1997). 
${ }^{17}$ S. J. Tans, M. H. Devoret, H. Dai, A. Thess, R. E. Smalley, B. L. Geeligs, and C. Dekker, Nature (London) 386, 474 (1997).

${ }^{18}$ M. Bockrath, D. H. Cobden, J. Lu, A. G. Rinzler, R. E. Smalley, L. Balents, and P. L. McEuen, Nature 397, 598 (1999).

${ }^{19}$ H. W. C. Postma, T. Teepen, Z. Yao, M. Grifoni, and C. Dekker, Science 293, 76 (2001).

${ }^{20}$ D. Bozovic, M. Bockrath, J. H. Hafner, C. M. Lieber, H. Park, and M. Tinkham, Appl. Phys. Lett. 78, 3693 (2001).

${ }^{21}$ M. J. Biercuk, N. Mason, J. M. Chow, and C. M. Marcus, Nano Lett. 4, 2499 (2004).

${ }^{22}$ P. Jarillo-Herrero, S. Sapmaz, C. Dekker, L. P. Kouwenhoven, and H. S. J. van der Zant, Nature 429, 389 (2004).

${ }^{23}$ K. Ishibashi, M. Suzuki, T. Ida, and Y. Aoyagi, Appl. Phys. Lett. 79, 1864 (2001).

${ }^{24}$ H. Li, Q. Zhang, and J. Li, Appl. Phys. Lett. 88, 013508 (2006).

${ }^{25}$ P. Stokes and S. I. Khondaker, ACS Nano 4, 2659 (2010).

${ }^{26}$ P. Stokes and S. I. Khondaker, Appl. Phys. Lett. 92, 262107 (2008).

${ }^{27}$ T. Hertel, R. E. Walkup, and P. Avouris, Phys. Rev. B 58, 13870 (1998).

${ }^{28}$ W. J. Liang, M. P. Shores, M. Bockrath, J. R. Long, and H. Park, Nature 417, 725 (2002).

${ }^{29}$ P. Stokes and S. I. Khondaker, Appl. Phys. Lett. 96, 083110 (2010).

${ }^{30}$ K. J. Kormondy, P. Stokes, and S. I. Khondaker, Nanotechnology 22, 415201 (2011)

${ }^{31}$ M. R. Islam, K. J. Kormondy, E. Silbar, and S. I. Khondaker, Nanotechnology 23, 125201 (2012).

${ }^{32}$ S. I. Khondaker, IEE Proc.: Circuits Devices Syst. 151, 457 (2004).

${ }^{33}$ P. Stokes and S. I. Khondaker, Nanotechnology 19, 175202 (2008).
${ }^{34}$ P. Stokes, E. Silbar, Y. M. Zayas, and S. I. Khondaker, Appl. Phys. Lett. 94, 113104 (2009).

${ }^{35}$ D. Joung, A. Chunder, L. Zhai, and S. I. Khondaker, Nanotechnology 21, 165202 (2010).

${ }^{36}$ M. R. Islam, D. Joung, and S. I. Khondaker, New J. Phys. 13, 035021 (2011).

${ }^{37}$ D. Joung, L. Zhai, and S. I. Khondaker, Phys. Rev. B 83, 115323 (2011).

${ }^{38}$ B. K. Sarker, S. Shekhar, and S. I. Khondaker, ACS Nano 5, 6297 (2011).

${ }^{39}$ B. K. Sarker, M. R. Islam, F. Alzubi, and S. I. Khondaker, Mater. Express 1, 80 (2011).

${ }^{40}$ See http://www.brewerscience.com for the physical properties of the SWNT solution.

${ }^{41}$ S. Shekhar, P. Stokes, and S. I. Khondaker, ACS Nano 5, 1739 (2011).

${ }^{42}$ C. Zhou, J. Kong, and H. Dai, Phys. Rev. Lett. 84, 5604 (2000).

${ }^{43}$ R. Hanson, L. P. Kouwenhoven, J. R. Petta, S. Tarucha, and L. M. K. Vandersypen, Rev. Mod. Phys. 79, 1217 (2007).

${ }^{44}$ J. Nygard, D. H. Cobden, M. Bockrath, P. L. McEuen, and P. E. Lindelof, Appl. Phys. A: Mater. Sci. Process. 69, 297 (1999).

${ }^{45}$ J. Park and P. L. McEuen, Appl. Phys. Lett. 79, 1363 (2001).

${ }^{46} \mathrm{H}$. Li and Q. Zhang, Appl. Phys. Lett. 94, 022101 (2009).

${ }^{47}$ J. Kong, C. Zhou, E. Yenilmez, and H. Dai, Appl. Phys. Lett. 77, 3977 (2000).

${ }^{48}$ P. L. McEuen, L. P. Kouwenhoven, S. Jauhar, J. Orenstein, K. McCormick, D. Dixon, Y. U. Nazarov, N. C. V. D. Vaart, and C. T. Foxon, Nanotechnology 7 , 406 (1996).

${ }^{49}$ M. Bockrath, W. Liang, D. Bozovic, J. H. Hafner, C. M. Lieber, M. Tinkham, and H. Park, Science 291, 283 (2001). 